\title{
A Radiation Tolerant Phototube Power Supply for the Electromagnetic Calorimeter of the HERA- $B$ Experiment.
}

\author{
G. Avoni ${ }^{\text {a }}$, C. Baldanza ${ }^{\text {a }}$, M. Bargiotti ${ }^{a}$, A. Bertin ${ }^{\text {a }}$, \\ B. Bobchenko ${ }^{\text {b }}$, M. Bruschi ${ }^{\mathrm{a}}$, M. Capponi ${ }^{\mathrm{a}}$, I. D'Antone ${ }^{\mathrm{a}}$, \\ S. De Castro ${ }^{\text {a }}$, L. Fabbri ${ }^{\text {a }}$, P. Faccioli ${ }^{\text {a }}$, B. Giacobbe ${ }^{\text {a }}$, \\ Yu. Gilitsky ${ }^{\mathrm{c}}$, A. Golutvin ${ }^{\mathrm{b}}$, O. Gouchtchine ${ }^{\mathrm{b}}, \mathrm{F}$. Grimaldi ${ }^{\mathrm{a}}$, \\ I. $\operatorname{Lax}^{a}$, I. Massa ${ }^{a}$, I. Matchikhilian ${ }^{b}$, S. Meneghini ${ }^{a}$, \\ Yu. Mikhailov ${ }^{\mathrm{c}}$, E. Novikov ${ }^{\mathrm{b}}$, M. Piccinini ${ }^{\mathrm{a}}$, M. Poli ${ }^{\mathrm{d}}$, \\ M. Rizzi ${ }^{\text {, }}$ V. Rusinov ${ }^{b}$, N. Semprini-Cesari ${ }^{a}$, S. Shuvalov ${ }^{\mathrm{e}}$, \\ A. Soldatov ${ }^{c}$, R. Spighi ${ }^{a}$, E. Tarkovsky ${ }^{b}$, M. Villa $^{\text {a }}$, A. Vitale ${ }^{\text {a }}$, \\ A. Zoccoli ${ }^{\text {a }}$, and M. Zuffa ${ }^{\text {a }}$ \\ ${ }^{a}$ Istituto Nazionale di Fisica Nucleare(INFN), Sezione di Bologna, Via Irnerio, \\ 46, I-40126 Bologna,Italy \\ ${ }^{\mathrm{b}}$ Institute of Theoretical and Experimental Physics, B. Cheremushkinskaya, 25, \\ Moscow 117259, Russia \\ ${ }^{\mathrm{c}}$ Institute of High Energy Physics, RU-142248, Protvino, Moscow region, Russia \\ ${ }^{\mathrm{d}}$ INN Sezione di Bologna and Dipartimento di Energetica "S. Stecco" Universitá \\ di Firenze, Italy \\ ${ }^{\mathrm{e}}$ Institut für Physik, Humboldt Universität zu Berlin, Newtonstr.15, D-12489 \\ Berlin, Germany ${ }^{1}$
}

\begin{abstract}
The design of the phototube power supply for the HERA- $B$ Electromagnetic Calorimeter is presented. A choice of the solution on the basis of the Cockcroft-Walton voltage multiplier is validated. Schematics developed are discussed. A special section describes the behaviour of the crucial components under irradiation since radiation tolerance becomes one of the most important items in the severe HERA- $B$ radiation environment. Finally performance achieved is presented.
\end{abstract}

Key words: HERA-B, calorimeter, shashlik, photomultiplier, Cockcroft, Walton PACS: $29.40 . \mathrm{Vj}$

Preprint submitted to Elsevier Science 


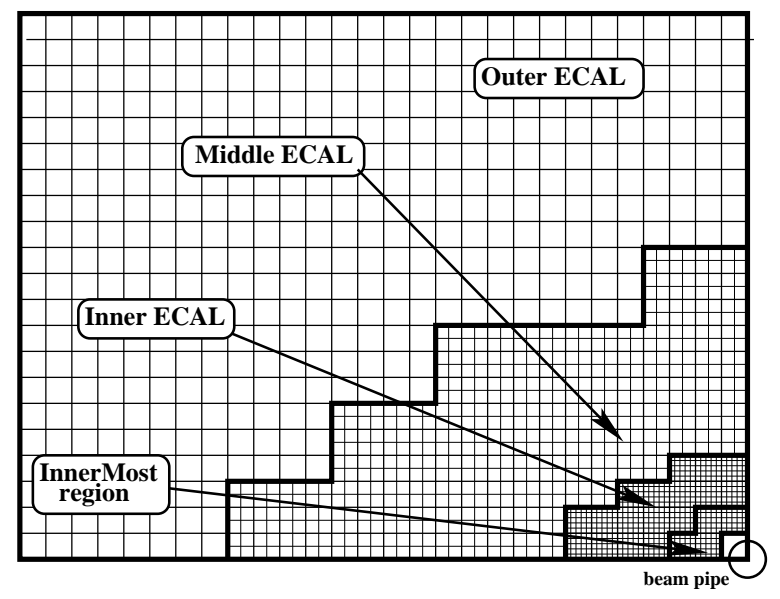

Fig. 1. The HERA-B ECAL schematic view Fig. 2. Distribution of the gain of (one of four symmetric quadrants)

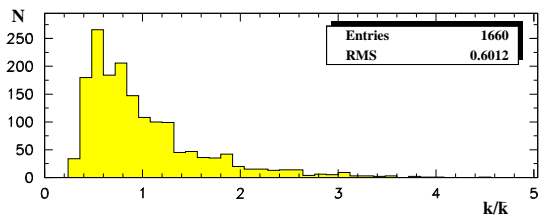

(a) FEU-68

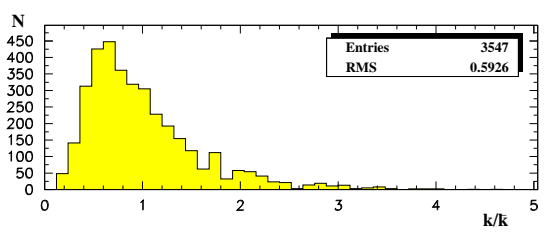

(b) FEU-84-3

\section{Introduction}

The HERA- $B$ Electromagnetic Calorimeter $(E C A L)[1]$ of the HERA- $B$ experiment [2] is based on shashlik technology [3]. The rectangular assembly (Fig. 1) comprises three sections (Inner, Middle and Outer ECALs), which are constructed from modules of the same transverse dimensions but different granularity in order to match the steep radial dependence of particle flux density. The light collected in each cell is read out by means of a photomultiplier (PM, phototube) [4]. The area close to the beam pipe (so called InnerMost region) is equipped with phototubes with radiation hard silica windows, while, in the rest of the $E C A L$, PMs with ordinary glass windows, manufactured in Russia, are used. The total number of readout channels is 5956.

The design of the phototube high voltage (HV) supply is dictated by the detector operating regime [2]. The general specifications are summarized in Table 1, additional comments are listed below:

(1) Radiation hardness. All the supply components must have sufficient radiation hardness, particularly those to be installed in the ECAL area near the beam pipe (InnerMost region), where the expected radiation dose could reach $15 \mathrm{kGy} /$ year;

(2) Dynamic range of output currents. The dynamic range of the readout ADC for PM response digitization is limited to 12 bits and $50 \mathrm{fC} /$ count, which corresponds to the maximum current amplitude of $10 \mathrm{~mA}$ for the expected PM pulse shape in the HERA- $B$ environment. The FEU-68 response becomes non-linear for anode current $\left(I_{\mathrm{A}}\right)$ amplitudes exceeding $1 \mathrm{~mA}$. Thus, for that type of PM, an additional intermediate amplifier

$\overline{1}$ supported by the Bundesministerium für Bildung und Forschung, FRG, under contract numbers 05-7BU35I, 05-HB1KHA 
Table 1

General specifications for the PM HV supply design. $I_{\mathrm{A}}-\mathrm{PM}$ anode current.

\begin{tabular}{|l|c|c|c|c|}
\hline & InnerMost & Inner & Middle & Outer \\
\hline PM type & R-5600 & FEU-68 & FEU-84-3 & FEU-84-3 \\
\hline $\begin{array}{l}\text { Max annual radiation dose } \\
\text { in PMs plane, kGy }\end{array}$ & 15 & 4 & 1 & 0.3 \\
\hline Average $I_{\mathrm{A}}, \mu \mathrm{A}$ & 60 & 4 & 60 & 80 \\
\hline Max pulse $I_{\mathrm{A}}, \mathrm{mA}$ & 10 & 1 & 10 & 10 \\
\hline Max PM gain $\times 10^{5}$ & 1.4 & 0.4 & 2.4 & 2.2 \\
\hline Max non-linearity, $\%$ & 2 & 2 & 2 & 2 \\
\hline Transverse cell size, cm & 2.23 & 2.23 & 5.58 & 11.15 \\
\hline
\end{tabular}

must be foreseen;

(3) Mean $I_{\mathrm{A}}$. The calorimeter must be able to function properly at severe occupancy conditions corresponding to target interaction rates up to $40 \mathrm{MHz}$ [2]. In particular, PMs must maintain their performance at the required level surviving mean anode currents up to an upper limit of $0.1 \mathrm{~mA}$;

(4) PM gain. The PMs employed show significant gain spread at the same value of PM high voltage supplied $\left(U_{\mathrm{HV}}\right)$, see Fig. 2. The HV supply design must provide the ability of regulating $U_{\mathrm{HV}}$ over a wide range in order to (i) compensate this effect, (ii) optimally exploit the detector dynamic range and (iii) match the requirement of pretrigger logic [5] to have the uniformity of the calorimeter channel response of the order of few percent. According to the project specifications, the ECAL energy resolution value should be kept below $2 \%$ for asymptotically high energy depositions. This puts an additional demand on the high stability of PM gain;

(5) Linearity of the output signal. The maximal non-linearity ${ }^{2}$ of the PM response at maximal $I_{\mathrm{A}}$ should not exceed $2 \%$;

(6) Heat dissipation. Due to the compact Inner section geometry, heat dissipation must be minimized unless a dedicated cooling system is foreseen;

(7) Noise. Noise from the supply must not spoil ADC pedestal width, whose typical value is comparable to one ADC count. The maximum permissible level of noise is defined thus by the ADC resolution;

2 Near the upper limit of ECAL PM pulse amplitudes range the response of FEU68/FEU-84-3 to a light signal starts to deviate from proportionality progressively as the anode currents increase. Henceforth, deviation of the PM response to large amplitude pulses from the linear extrapolation of the linear small input signal region will be referred as the non-linearity level. For numerical estimates it will be expressed as the percentage of the value predicted by extrapolation. 


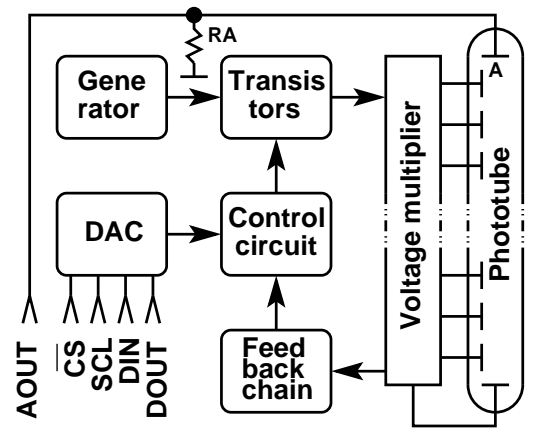

Fig. 3. PM HV supply block-scheme

(8) Other demands. Homogeneity, i.e. similar HV supply designs for all three calorimeter sections, is preferable in order to facilitate detector operation. Finally, component costs, printed circuit board (PCB) costs and production costs should be minimized.

The main disadvantages of the standard HV supply solution of a passive (resistive) divider in the HERA- $B E C A L$ environment are (i) large power dissipation and (ii) inability to assure proper PM operation without providing auxiliary power to the last dynodes. Mainly for these reasons, it cannot meet the requirements listed above without a significant increase of the cost per channel and a more complicated ECAL operation. Instead, the PM HV supply was developed on a basis of the Cockcroft-Walton voltage multiplier (C-W) [6], which has low power dissipation, is inexpensive, simple in exploitation and, as will be proven below, perfectly matches the purpose of keeping the PM performance within design specifications at the high pulse rates needed for HERA- $B$ operation.

\section{HV supply design}

The general block-scheme of the PM HV supply is presented in Fig. 3. It comprises the clock generator, the pumping transistors, the voltage multiplier itself, the feedback chain, the control circuit to trace the stability of the voltage supplied, and the DAC for digital HV control.

The simplified schematics of the HV supply is presented in Fig. 4. Three HV supply versions for three different types of phototubes are adopted. The dashed rectangles single out different chains, each with a caption designating the applicable supply type. Distinct features of the circuitry are summarized in Table 2.

The basic circuitry parameters are adjusted according to the following considerations: 

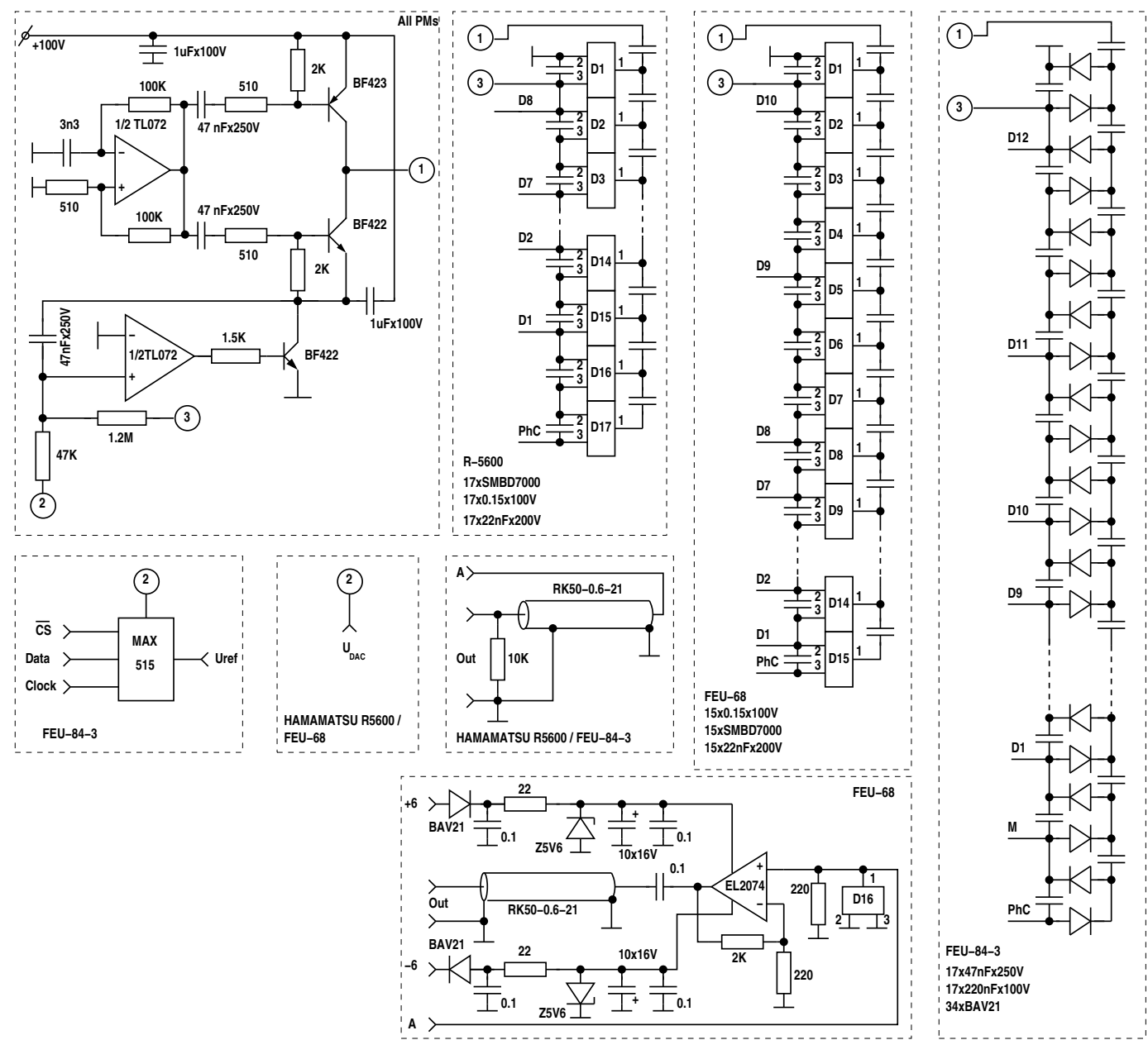

Fig. 4. Simplified schematics of the HV supply (see comments in the text).

Table 2

Special features of HV supply design for different phototubes. A designates anode, $\mathrm{D}_{i}$ - i-th dynode, where enumeration starts from cathode side, 1 denotes $U_{\text {stage }}$.

\begin{tabular}{|l|c|c|c|}
\hline & R-5600 & FEU-68 & FEU-84-3 \\
\hline Number of stages & 17 & 15 & 17 \\
\hline $\begin{array}{l}\text { Optimal voltage pattern } \\
\text { A-D }\end{array}-\mathrm{D}_{N-1}-\mathrm{D}_{N-2}-\mathrm{D}_{N-3}$ & $1-2-2-2$ & $1-3-3-1$ & $1-3-2-1$ \\
\hline$<U_{\mathrm{HV}}>, \mathrm{V}$ & 650 & 1300 & 1200 \\
\hline
\end{tabular}

(1) Gain. On the basis of laboratory tests the mean value of the HV supplied to the PM was fixed to be near $1 \mathrm{kV}$ to provide the required range of output signals (see Table 2).

(2) Linearity. An acceptably low non-linearity level in the required range of $I_{\mathrm{A}}$ was achieved by properly tuned potential configurations over the last 2 or 3 PM dynodes (see Table 2).

(3) The number of stages. A low number of stages (one-half period rectifiers 
in $\mathrm{C}-\mathrm{W}$ multiplier) is favourable for minimizing ripple and component count, but also results in increased potential drop per stage $U_{\text {stage }}$ and thus places more stringent demands on the voltage rating of components. The chosen value of $U_{\text {stage }}=100 \mathrm{~V}$ was a compromise between the size and quality of the components (diodes and capacitors) available and overall cost. This number also allows for a large spread of PM gains. $U_{\text {stage }}$ defines an overall circuit feeding voltage $V$ as well.

(4) Gain control. A digital implementation is chosen for PM voltage supplied control. Most of the commercially available digital-to-analog converters (DACs) with serial interface are not sufficiently radiation hard to operate in the Inner $E C A L$ area (see discussion below in the dedicated section). For that reason, the C-W circuitry for both the R-5600 and the FEU-68 does not include either the DAC or its reference voltage chain, which are instead part of the master voltage control board, and therefore are outside the irradiated area. In the case of the FEU-84-3 (Middle/Outer section), each C-W PCB is equipped with its own DAC, specifically with the inexpensive 10-bit MAX515 chip. The basic concept of the control chain is described in [7].

(5) The frequency of the pumping voltage. Besides the amplitude of the pumping pulser discussed above, the two most important parameters to be optimized are the frequency of the pumping voltage and the rise/fall time of pumping pulse edges. At fixed capacitor values, the minimum value of pumping frequency is defined by the ripple and consequently by the mean current through the multiplier circuit. Increasing $f$ would allow for smaller capacitor values, but would progressively increase pick-up noise in the output signal. The sharpening of the pulse edges somewhat connected with the increase of $f$ would also result in increased pick-up and an objectionable sharp non-linear increase in power consumption due to the forward currents in switching transistors at times when one is already in the on state and the second is not yet in the off state. Such an effect can be eliminated by proper adjustment of rise/fall time of pumping pulse edges. The value of pumping frequency then is defined as a compromise between (i) dimensions, quality, size and commercial availability of the components (ii) pick-up level requirements (see Section 1) and (iii) minimization of circuit power consumption. Taking into account these considerations, the value of $f$ was finally fixed to $30 \mathrm{kHz}$. Some additional discussion can be found in [8].

(6) Components.

- Multiplier circuits. In order to minimize the ripple, capacitors of maximal available capacity and with minimal leakage current are preferable, while diodes should have minimal reverse currents. In the PM supplies utilized in the Inner section, SMD-components (diodes and capacitors) are employed because of the small size of the PCB.

- Radiation resistance (see details in the next section). The most important radiation-related issue is the behaviour of active components under 
irradiation at dosage levels of up to a few tens of $\mathrm{kGy}$, while passive elements are well known to be sufficiently radiation hard. For that reason the supply design uses a minimum number of active components and seeks to ensure that the circuit is insensitive to the degradation of their crucial parameters.

- The precision of the output voltage reproduction is defined by the tolerances of the DAC reference and the resistors of the feedback chain. For that reason, critical components are selected to have $1 \%$ tolerance and the same temperature coefficient of resistance (TCR). The temperature dependence then could be neglected since it is defined by the ratio of the resistances.

(7) Other considerations. According to the R-5600 photomultiplier data sheet [9], the maximum value of the voltage supplied is $1000 \mathrm{~V}$. This limit is enforced in the software of the common ECAL monitoring and control system rather than in the supply circuitry itself, in order to support better HV-supply system homogeneity;

To achieve the required range of output signals an additional preamplifier, based on the EL2074 operational amplifier, is introduced into the circuitry of the FEU-68 supply. The EL2074 utilizes fast BJT technology and is sufficiently radiation hard to operate in the calorimeter area equipped with these phototubes.

Photographs of the HV supplies coupled with the different types of phototubes are shown in Fig. 5.

\section{Radiation hardness of components}

The measurements of the radiation resistance of the components were carried out using the $70 \mathrm{GeV}$ proton beam of the U70 accelerator (IHEP, Protvino, Russia). A detailed description of the experimental setup can be found in [10]. Tested samples were irradiated using secondaries from the internal Be target. An auxiliary ionization chamber located downstream of the tested samples served for on-line dose monitoring. A precise determination of the accumulated dose was performed off-line with the help of thin-film dosimeters [11] glued directly onto the samples.

- The crucial parameters of the components of the rectifier stages are the capacitance and the voltage rating of the capacitors; the leakage current and the reverse voltage of the diodes. All of these parameters were found to be stable at doses of up to a few tens of $\mathrm{kGy}$ for the chosen components, proving their suitability for the HERA- $B$ environment.

- For a few types of bipolar high voltage transistors (BF422(423), FMMT493(593), FMMT497(597) and PMBT5401(5551)) the degradation of the most crucial 


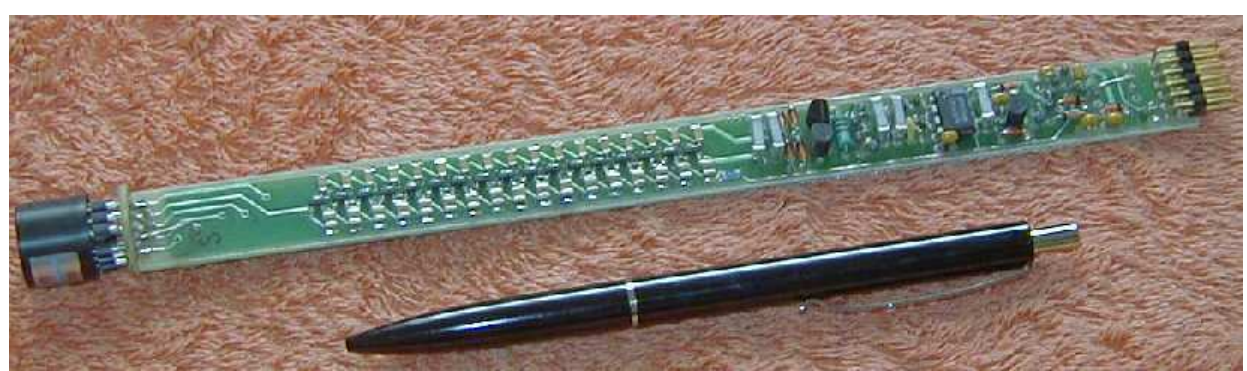

(a) HAMAMATSU R-5600

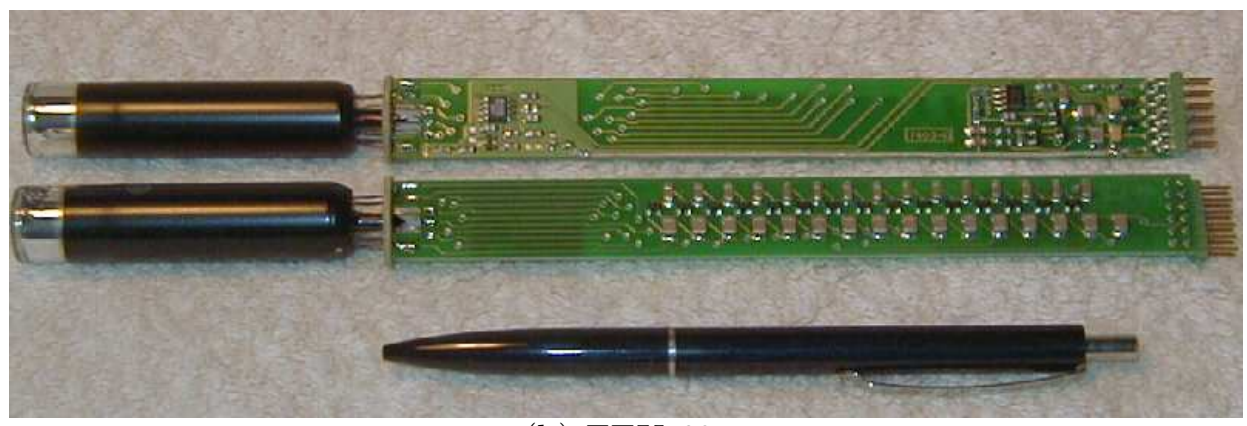

(b) FEU-68

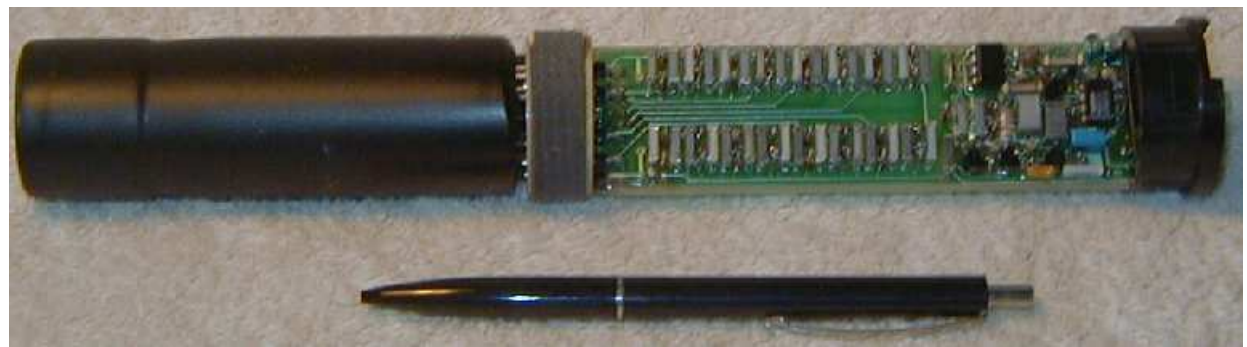

(c) FEU-84-3

Fig. 5. Phototubes instrumented with HV supplies.

parameter, the current gain $\mathrm{h}_{f e}$, with accumulated dose has been investigated. At the maximal dose $(\sim 20 \mathrm{kGy})$ tested, the value of $\mathrm{h}_{f e}$ was found to be in the range from 1 to 10 for all transistors tested compared to initial values in the range from 70 to 180 . Nonetheless, it is still sufficient for C-W applications, where transistors mainly serve as switches for rail-to-rail operation.

- Generator and control circuits are built on the basis of the double operational amplifier. The first amplifier section, with positive feedback, is an oscillator used for driving the switching transistors. The second one is a comparator. Therefore, the open loop gain and bandwidth are most critical for C-W operation. For the TL072, which is produced with FET-BJT technology, open loop gain shows no significant change for doses of up to 17-20 kGy, while the pumping frequency slowly degrades: it was measured to be $20 \mathrm{kHz}$ at a dose of $10 \mathrm{kGy}$ compared to an initial value of $30 \mathrm{kHz}$. At the same time the overall performance of a C-W with an installed TL072 show no visible degradation up to doses of 17-20 kGy. With further irradiation, TL072 becomes fully damaged: C-W output voltage drops to zero and 

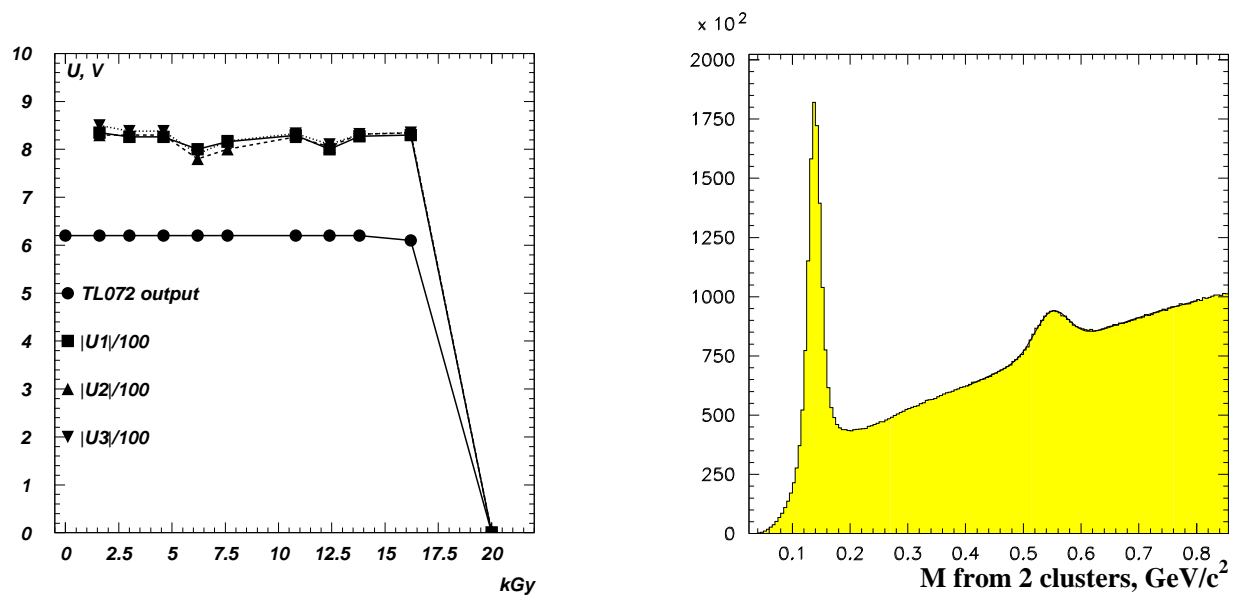

Fig. 6. Irradiation test of TL072 (see com- Fig. 7. Two ECAL clusters invariant ments in the text of the article). mass. $\pi^{0}$ and $\eta$ peaks are clearly seen.

the power consumption of the supply significantly decreases. The TL072 behaviour under irradiation is illustrated in Fig. 6. During tests the gains of both amplifiers were set to 100 to probe the lower limit, where the circuit is still operational. One amplifier was fed with the positive, while the other - with negative voltage $(6 \mathrm{~V})$. The filled circles represent the voltage difference from different sign outputs, other markers (squares and triangles) indicate the voltage from the second $\mathrm{C}-\mathrm{W}$ stage. In total, three kinds of C-W, all exploiting the TL072 operational amplifier but with different types of transistors and diodes, were studied.

- The last active component is a DAC. Nearly all commercially available DACs with serial interfaces are built with CMOS technology and consequently they are not sufficiently radiation hard to survive the InnerMost $E C A L$ region environment. In particular, the MAX515 proved to operate reliably only up to $\sim 3 \mathrm{kGy}$, whereupon it started to show functional failure losing the ability to control the output voltage, which is maintained at zero level independently of input code.

\section{Quality control and performance}

Quality control. All PM supplies have been checked at least twice with a dedicated test setup. The procedure included the measurement of voltages on all PM electrodes at the maximum DAC input code and nominal HV. The values of the currents consumed from all power sources were checked to be tolerable. Afterwards, all PCBs were coated with a special protective spray and coupled with the PMs. Each doublet 'PM+C-W' was tested in the laboratory in order to pre-calibrate the gain of the phototube and measure significant parame- 
ters, particularly the non-linearity level at maximal anode current amplitude. Doublets with proper non-linearity level were accepted for installation in the $E C A L$.

Performance.

- The current consumption of the C-W circuit itself (without PM connected), from a $100 \mathrm{~V} \mathrm{HV}$ source, does not exceed $0.2 \mathrm{~mA}$, which is smaller by an order of a magnitude with respect to a simple passive divider.

- The dispersion of the $U_{\text {stage }}$ values over the half period of the pumping voltage does not exceed $0.1 \%$ at a mean level of consumption of about $1 \mathrm{~mA}$. This is equivalent to a gain variation of less than $2 \%$, thus demonstrating the ability of $\mathrm{C}-\mathrm{W}$ to keep stable dynode voltages at rates up to several $\mathrm{MHz}$ of large amplitude pulses.

- To quantify reproducibility, a standard PM was tested with a large number of C-W supplies operated at a fixed DAC setting and with a constant input illumination from a LED. The RMS variation of signals was found to be less than $2 \%$.

- The chosen value of feeding voltage, $V$, allows to regulate PM HV supplied up to $1700 \mathrm{~V}$, which easily accommodates a large PM gain spread and possible module light yield degradation in the InnerMost region. The precision of the PM gain control is within 1\%, thus proving that a 10-bit DAC range is sufficient to fulfill $E C A L$ design specifications.

- The mean and RMS values of non-linearity levels over all doublets 'PM+C-W' which have been installed in the ECAL, are $-0.24 \%$ and $0.59 \%$ for FEU-68; $-0.22 \%$ and $0.64 \%$ for FEU-84-3 PMs. All R-5600 PMs have linear responses in the full dynamic range of current amplitudes. The numbers obtained fulfill project requirements even with some margin.

- During the full HERA- $B$ lifetime, the rate of malfunctions did not exceed $0.1 \%$ per month. At the end of the data taking in 2003, after more than a year of $E C A L$ operation in "not allowed to touch" conditions the number of channels with improperly working bases did not exceed $1.3 \%$.

- The cost of the PM supply system is dominated by the development and production expenses. The component kit itself counts for approximately 10 Euro/PM unit.

\section{Conclusions}

The HERA- $B E C A L$ phototube HV supply system was developed on the basis of the Cockcroft-Walton multiplier. It operated successfully during the 4 years of the HERA- $B$ detector running period without serious faults or performance loss. All the relevant system parameters were proven to be well within design specifications. The contribution of PM supply noise to the energy and spatial 
resolution was negligible [12]. Figure 7 represents the invariant mass of two $E C A L$ clusters with common transverse energy $e_{T}>2 \mathrm{GeV}$. Two peaks centered near the mass values of $\pi^{0}$ and $\eta$ mesons are clearly seen. The $\eta$ mass resolution is $30 \mathrm{MeV}$.

Due to a revised physical program [13], HERA- $B$ detector operated under only moderate radiation environment being compared with Technical Design Report [2]. The net dose received by the PM supplies closest to the beam pipe is $\sim 18 \mathrm{kGy}$. No degradation of overall supply performance, caused by the radiation damage of components, was observed.

\section{Acknowledgements}

Authors are very grateful to the DESY laboratory for the strong support since the beginning of the HERA- $B$ experiment. They also would like to express sincere thanks to all of our colleagues from the HERA- $B$ collaboration for useful discussions and suggestions. The authors would like to thank M. Medinnis (DESY) for critical reading of this manuscript.

\section{References}

[1] G. Avoni et al., Nucl. Instr. and Meth. A461 (2001) 332.

[2] E. Hartouni et al., HERA-B: Design Report, DESY-PRC 95/01.

[3] G.S. Atoyan et al., Nucl. Instr. and Meth. A320 (1992) 144 .

[4] (DESY), HERA-B Proposal, DESY-PRC 94/02.

[5] C. Baldanza et al., Nucl. Instr. and Meth. A409 (1998) 643.

[6] H.Greinacher, Erzeugung einer Gleichspannung vom vielfachen Betrage einer Wechselspannung ohne Transformator, Bulletin des Schweiz. Elektrotechn. Vereins 11 (1920), H.Greinacher, Zeitschrift f. Physik 4 (1921), J.D.Cockcroft and E.T.S.Walton, Proc. Roy. Soc. A136 (London, 1932) 619, J.D.Cockcroft and E.T.S.Walton, Proc. Roy. Soc. A137 (London, 1932) 229.

[7] L.Hubbeling, A low power, high frequency DC-DC converter for generating high voltages, EP Internal report 78-5 (Geneva, 1978).

[8] I. D'Antone et al., Nucl. Instr. and Meth. A480 (2002) 555.

[9] HAMAMATSU Photonics Photomultiplier Tube R-5600 Series. 
[10] Alexandrov L.M. et al., Radiation hardness of some elements and the electronic modules of equipment on accelerators, IHEP preprint 2002-25 (Protvino, 2002).

[11] A. V. Antipov et al., Precise densitometer for thin-film dosimeters, Nucl. Instr. and Meth. B94 (1994) 338.

[12] G. Avoni et al., The Electromagnetic Calorimeter of the HERA-B experiment, in: C. Cecchi, P.Cenci, P. Lubrano and M. Pepe, eds., CALORIMETRY IN PARTICLE PHYSICS, Proceedings of the Eleventh International Conference (World Scientific, 2005) 27-32.

[13] (DESY), HERA-B: report on status and prospects. Executive summary, DESY$P R C$ 00/04 